\title{
A COUNTABLY COMPACT, FIRST COUNTABLE, NONNORMAL $T_{2}$-SPACE
}

\section{J. E. VAUGHAN}

\begin{abstract}
We construct a space which has the properties listed in the title and which is also locally compact, locally countable, zero-dimensional, $\omega$-bounded and has cardinality $k_{1}$.
\end{abstract}

1. Introduction. The fact that every countably compact, first countable, $T_{2}$-space is regular is a result which is well known and easy to prove. It is a natural question to ask if every such space is also normal. We first heard of this question in 1969 when it was raised in a conversation by $R$. M. Stephenson, Jr. He sent this question to Mary Ellen Rudin, and she sent back an example of a countably compact, first countable, nonnormal $T_{2}$-space. Since her example has never been published, we take this opportunity to mention that its construction was based on the techniques in [4], and in particular, the construction made use of the continuum hypothesis $(\mathrm{CH})$. Professor Rudin conjectured in her letter that such an example could be constructed without using any set-theoretic assumptions beyond the usual axioms of set-theory including the axiom of choice (ZFC). The example which we construct in this paper shows that her conjecture is correct.

Several other countably compact, first countable, nonnormal $T_{2}$-spaces have been constructed recently. A. Ostaszewski (using the set-theoretic assumption $\diamond$ ) [3] constructed such a space which is, in addition, hereditarily separable. M. Wage (also using $\diamond$ ) [5] constructed one which is hereditarily separable and perfect. M. Dahroug (using Martin's Axiom plus not $\mathbf{C H}$ ) constructed one which is separable (unpublished), and W. Weiss also constructed separable ones under several other set-theoretic assumptions (unpublished). It should be noted that Rudin's example (using $\mathrm{CH}$ ) is separable.

In this paper, we construct within ZFC a space having the properties listed in the title and abstract. Since this space is $\omega$-bounded (i.e., every countable subset has compact closure) it is not separable. It follows from the preceding discussion that a natural question to raise now is this: Does there exist within ZFC a countably compact, first countable, nonnormal, separable, $T_{2}$-space?

2. The example. We first establish some notation. Let $\omega$ denote the set of finite ordinals and let $\omega_{1}$ denote the set of countable ordinals. Put

Presented to the Society, January 25, 1979; received by the editors July 13, 1978. AMS (MOS) subject classifications (1970). Primary 54D15, 54A25; Secondary 02K25. Key words and phrases. Countably compact, first countable, nonnormal, separable. 


$$
\begin{aligned}
& \Lambda=\left\{\lambda<\omega_{1}: \lambda \text { is a limit ordinal }\right\}, \\
& A=\Lambda \cup\{\lambda+2 n: \lambda \in \Lambda \cup\{0\} \text { and } 1<n<\omega\}, \text { and } \\
& B=\Lambda \cup\{0\} \cup\{\lambda+2 n-1: \lambda \in \Lambda \cup\{0\} \text { and } 1<n<\omega\} .
\end{aligned}
$$

The set $X$ on which we will define the desired topology is the subset of $\omega_{1} \times(\omega+2)$ defined by $X=\left(\omega_{1} \times \omega\right) \cup H \cup K$ where $H=\left\{h_{\alpha}=(\alpha, \omega)\right.$ : $\alpha \in A\}$ and $K=\left\{k_{\alpha}=(\alpha, \omega+1): \alpha \in B\right\}$. The sets $H$ and $K$ will be used to show that the space is not normal.

The construction of the topology $T$ on $X$ is by transfinite induction, and is indebted to the constructions of Ostaszewski and Wage mentioned in $\S 1$. The main successor ordinal step is given in the following result.

LEMMA. Let $Z$ be a set, and $S$ a $T_{2}$-topology on $Z$ which has a countable base consisting of compact-open sets. Let $H, K$ be disjoint closed subsets of $Z$, and $p$, $q$ two points not in $Z$. Then there exists a topology $T$ on $Y=Z \cup\{p\} \cup\{q\}$ such that

(a) $(Z, S)$ is an open subspace of $(Y, T)$;

(b) $(Y, T)$ is a compact, $T_{2}$-space having a countable base of compact-open sets;

(c) $H \cup\{p\}$ and $K \cup\{q\}$ are (disjoint) closed sets in $(Y, T)$.

Proof. Since $Z$ has a countable base and is zero dimensional, we have $\operatorname{Ind}(Z)=0$ [1, Chapter II, §2.E.]. Thus, there exist disjoint clopen sets $U, V$ in $Z$ such that $Z=U \cup V, H \subset U$, and $K \subset V$. Let $\left\{B_{i}: i<\omega\right\}$ be a countable base of compact-open sets of $Z$. Define for $n<\omega$

$$
\begin{aligned}
& V(p, n)=\{p\} \cup\left(U \backslash \bigcup_{i<n} B_{i}\right), \quad \text { and } \\
& V(q, n)=\{q\} \cup\left(V \backslash \bigcup_{i<n} B_{i}\right) .
\end{aligned}
$$

Let $T$ be the topology on $Y$ having as a base $S \cup\{V(p, n): n<\omega\} \cup$ $\{V(q, n): n<\omega\}$. The space $(Y, T)$ is a two-point compactification of $(Z, S)$ satisfying the properties (a), (b) and (c).

Construction of the example. For $\alpha<\omega_{1}$, put

$$
\begin{aligned}
& H_{\alpha}=\left\{h_{\beta}: \beta \in A \text { and } \beta<\alpha\right\}, \\
& K_{\alpha}=\left\{k_{\beta}: \beta \in B \text { and } \beta<\alpha\right\}, \text { and } \\
& X_{\alpha}=(\alpha \times \omega) \cup H_{\alpha} \cup K_{\alpha} .
\end{aligned}
$$

Assume for $\alpha<\gamma$ (where $\gamma<\omega_{1}$ ) we have defined topologies $T_{\alpha}$ on $X_{\alpha}$ such that the following hold for all $\beta<\alpha<\gamma$.

(1) $\left(X_{\beta}, T_{\beta}\right)$ is a open subspace of $\left(X_{\alpha}, T_{\alpha}\right)$.

(2) $\left(X_{\alpha}, T_{\alpha}\right)$ is a $T_{2}$-space having a countable base of compact-open sets, and further if $\alpha$ is a successor ordinal, then $\left(X_{\alpha}, T_{\alpha}\right)$ is compact.

(3) $H_{\alpha}$ and $K_{\alpha}$ are closed (disjoint) sets in $\left(X_{\alpha}, T_{\alpha}\right)$.

(4) If $\lambda+2 n<\alpha$ and $\lambda$ is a limit ordinal or $\lambda=0$ and $1<n<\omega$, then the 
sequence $\{(\lambda+2 n, i): i<\omega\}$ converges to $h_{\lambda+2 n}$ in $\left(X_{\alpha}, T_{\alpha}\right)$ and if $\lambda+2 n-$ $1<\alpha$, then the sequence $\{(\lambda+2 n-1, i): i<\omega\}$ converges to $k_{\lambda+2 n-1}$ in $\left(X_{\alpha}, T_{\alpha}\right)$.

(5) $(\alpha \times \omega)$ with the product topology (where $\alpha$ and $\omega$ have the order topology) is an open subspace of $\left(X_{\alpha}, T_{\alpha}\right)$.

We construct $\left(X_{\gamma}, T_{\gamma}\right)$.

Case 1. $\gamma$ is a limit ordinal. Put $T_{\gamma}=\left\{U \subset X_{\gamma}: U \cap X_{\alpha} \in T_{\alpha}\right.$ for all $\alpha<\gamma\}$. Then $\left(X_{\gamma}, T_{\gamma}\right)$ is the direct limit of the subspaces $\left(X_{\alpha}, T_{\gamma}\right)$, and (1)-(5) are easily verified for $\alpha=\gamma$.

Case 2. $\gamma=\lambda+1$ where $\lambda$ is a limit ordinal.

For each $(\lambda, i)$ where $i<\omega$ and for each $\beta<\lambda$, define $V((\lambda, i), \beta)=(\beta, \lambda]$ $\times\{i\}$. In order to define $T_{\gamma}$, we define an intermediate space $(Y, S)$ as follows. Set $Y=X_{\lambda} \cup\{(\lambda, i): i<\omega\}$ and let $S$ be the topology on $Y$ having as a base $S \cup\{V((\lambda, i), \beta): i<\omega, \beta<\lambda\}$. Since $(\lambda \times \omega)$ is an open subspace of $\left(X_{\lambda}, T_{\lambda}\right)$, we see that each $V((\lambda, i), \beta) \cap X_{\lambda}=(\beta, \lambda) \times\{i\} \in T_{\lambda}$. Thus $\left(X_{\lambda}, T_{\lambda}\right)$ and $(\lambda+1) \times \omega$ are open subspaces of $(Y, S)$ and $H_{\lambda}$ and $K_{\lambda}$ are closed sets in $(Y, S)$. Now $X_{\gamma}=Y \cup\left\{h_{\lambda}\right\} \cup\left\{k_{\lambda}\right\}$. Let $T_{\gamma}$ be the topology on $X_{\gamma}$ given by Lemma 1 where $H=H_{\lambda}, K=K_{\lambda}, p=h_{\lambda}$ and $q=k_{\lambda}$. So $\left(X_{\gamma}, T_{\gamma}\right)$ is a compact $T_{2}$-space having a countable base of compact-open sets and $H_{\gamma}, K_{\gamma}$ are closed (disjoint) in $\left(X_{\gamma}, T_{\gamma}\right)$. Thus (1), (2), (3) and (5) hold, and there is nothing new to check for (4).

Case 3. $\gamma=\lambda+2 n$ where $\lambda$ is a limit ordinal and $1<n<\omega$.

Note that $X_{\gamma} \backslash X_{\gamma-1}=\{(\lambda+2 n-1, i): i<\omega\} \cup\left\{k_{\lambda+2 n-1}\right\}$. Thus we need only define neighborhoods for these points. Define $T_{\gamma}$ so that $\{(\lambda+2 n$ $-1, i): i<\omega\}$ is a sequence of isolated points which converges to $k_{\lambda+2 n-1}$. To do this put $V\left(k_{\lambda+2 n-1}, i\right)=\left\{k_{\lambda+2 n-1}\right\} \cup\{(\lambda+2 n-1, j): i<j<\omega\}$ for all $i<\omega$, and let $T_{\gamma}$ be the topology on $X_{\gamma}$ having as a base $T_{\lambda+2 n-1} \cup$ $\left\{V\left(k_{\lambda+2 n-1}, i\right): i<\omega\right\} \cup\{(\lambda+2 n-1, i): i<\omega\}$. Properties (1)-(5) are easy to check for $\alpha=\gamma$ because $\left(X_{\gamma}, T_{\gamma}\right)$ is the disjoint union of $\left(X_{\gamma-1}, T_{\gamma-1}\right)$ and a convergent sequence. (Also note that $h_{\lambda+2 n-1}$ is not defined; so there is nothing to do for $H$ at this step.)

Case 4. $\gamma=\lambda+2 n+1$ where $1 \leqslant n<\omega$. Proceed as in Case 3 except make $\{(\lambda+2 n, i): i<\omega\}$ a sequence of isolated points which converges to $h_{\lambda+2 n}$.

Define $T_{\omega_{1}}$ as in the limit ordinal case. Then $T=T_{\omega_{1}}$ is a topology on $X$. We check that the space $(X, T)$ has the desired properties. Since $\cup\left\{T_{\alpha}\right.$ : $\left.\alpha<\omega_{1}\right\}$ is a base for $T$, it is clear that $(X, T)$ is a first countable zero dimensional $T_{2}$-space. For each $\alpha<\omega_{1}, X_{\alpha+1}$ is a compact-open subspace of $X$. This implies that $X$ is locally compact, locally countable and $\omega$-bounded (since each countable subset of $X$ is contained in some $X_{\alpha+1}$ ).

To show that $X$ is not normal it suffices to show that if $U$ and $V$ are open sets in $X$ such that $U \supset H$ and $V \supset K$ then $\bar{U} \cap \bar{V} \neq \varnothing$.

Since $\{(\lambda+2 n, i): i<\omega\}$ converges to $h_{\lambda+2 n}$ for all limit ordinals $\lambda$, there 
exist $n_{0}<\omega$ and an uncountable set $F_{0} \subset \omega_{1}$ such that if $\alpha \in F_{0}$ and $i>n_{0}$ then $(\alpha, i) \in U$. Similarly for $V$, there exist $n_{1}<\omega$ and an uncountable set $F_{1} \subset \omega_{1}$ such that if $\alpha \in F_{1}$ and $i \geqslant n_{1}$, then $(\alpha, i) \in V$. Pick a sequence of countable ordinals from $F_{0} \cup F_{1}$ such that $\alpha_{0}<\alpha_{1}<\ldots<\alpha_{n}<\ldots$, and such that $\alpha_{i} \in F_{0}$ if and only if $i$ is an even integer. Define $\alpha=\sup \left\{\alpha_{i}\right.$ : $i<\omega\}$, and $n=\max \left\{n_{0}, n_{1}\right\}$. Then $(\alpha, n) \in \bar{U} \cap \bar{V}$ since $(\alpha, n)$ has its usual neighborhoods in $\omega_{1} \times \omega$ by property (5). This shows that $(X, T)$ is not normal.

REMARK. If we apply the construction of F. B. Jones [2] to the space $(X, T)$ we get a countably compact, first countable, $T_{2}$-space which is not completely regular.

\section{REFERENCES}

1. W. Hurewicz and H. Wallman, Dimension theory, Princeton Univ. Press, Princeton, N. J., 1948.

2. F. Burton Jones, Hereditarily separable, non-completely regular spaces, Topology Conf., Virginia Polytech. Inst. and State Univ., Lecture Notes in Math., no. 375, Springer-Verlag, Berlin and New York, 1974, pp. 149-152.

3. A. J. Ostaszewski, A countably compact, first countable, hereditarily separable, regular space which is not completely regular, Bull. Acad. Polon. Sci. Sér. Sci. Math. Astronom. Phys. 23 (1975), 431-435.

4. M. E. Rudin, A technique for constructing examples, Proc. Amer. Math. Soc. 16 (1965), $1320-1323$.

5. M. Wage, Countable paracompactness, normality, and Moore spaces, Proc. Amer. Math. Soc. 57 (1976), 183-188.

Department of Mathematics, University of North Carolnna at Greensboro, GreensBORO, NORTH CAROLINA 27412 